\title{
Study of Various Vesiculobullous Lesions Using Tzanck Smear
}

\author{
Sanchit Singhal and Hemalata $M^{*}$
}

Dept of Pathology, Kempegowda Institute of Medical Sciences, Bengaluru, India

\begin{abstract}
Background: The vesiculobullous reaction pattern is characterized by the presence of vesiclesor bullae within the epidermis or at the dermoepidermal junction. Despite some having characteristic presentations, it's difficult to make a definitediagnosis clinically. Hence, cytological evaluation is required for reliable and early diagnosis.Objectives of the study are to determine the incidence of various vesiculobullous lesions and evaluate cytology as a tool for early diagnosis of vesiculobullous lesions.

Methods: For Tzanck smears fresh vesicle or bulla was selected and incised with scalpel, reflectingthe roof of bulla. Base of the blister scraped gently and material spread on a glassslide. These smears were stained with MGG (air dried), Pap and H\&E stains (fixed).Skin punch biopsies from the bullae were subjected to routine fixation, processing,sectioning and staining.

Results: A total of 62 Tzanck smears were done for vesiculobullous lesions of skin of which 29had histopathological correlation. Herpes constitutedthe most common vesiculobullous disorder (42\%) followed by bullous pemphigoid (27.4\%)and pemphigus vulgaris (19.3\%). Most patients were in the age group 61- 70 years. TheM:F ratio of 1:1.38 showing female preponderance. Tzanck smears showed acantholyticcells in pemphigus group, eosinophils in bullous pemphigoid and multinucleate giantcells in viral blisters. Histopathology showed intraepidermal acantholysis inpemphigus vulgaris, subcorneal blister in pemphigus foliaceus and subepidermal inbullous pemphigoid.
\end{abstract}

Conclusion: Cytohistopathological correlation showed an overall sensitivity of $79 \%$. Tzanck smearshowed $96 \%$ sensitivity for viral infections. Tzanck smear is a quick, non-invasivemethod for the early diagnosis of vesiculobullous disorders.

Keywords: Vesicle; Bulla; Cytology; Acantholysis

\section{Introduction}

Cytology is the study of intrinsic characteristics and functions of individual cells. It was first used in the diagnosis of cutaneous disorders by Tzanck in 1947, particularly for herpes simplex. ${ }^{[1]}$ Since then this technique has found usage by dermatologists and pathologists for diagnosing various cutaneous dermatoses. ${ }^{[2-6]}$

Tzanck smear is a simple, rapid and reliable tool that can be used as a routine investigation in common vesiculobullous disorders, certain cutaneous infections such as chicken pox and in various genodermatoses. While the cytological findings are diagnostic in some lesion, in others they are only suggestive, requiring histopathological confirmation. ${ }^{[7]}$

Not many studies exist determining the utility of Tzanck smear as a diagnostic tool. Hence, the purpose of this study was to assess the cytomorphological features and evaluate cytology as a rapid and reliable method for early diagnosis of various cutaneous lesions.

\section{Materials \& Methods}

The study included 62 patients presenting with vesicles, pustules, bullae, papules, erosions and crusted lesions referred from the Department of Dermatology to the Department of Pathology, over a period of 15 months.

The lesions were cleaned, the peripheral portion of the blister was deroofed and the roof reflected. Excess fluid was gently removed by bloating and base of the blister was gently but thoroughly scraped. The cellular material was spread smoothly without scrubbing onto at least two clear slides. Dry fixed smear was stained with MGG stain and wet alcohol fixed smears with Pap and H\&E stain. Skin scraping was done for superficial erosions. For crusted lesions, these crusts were carefully removed with sterile forceps, and the base of the lesions was scraped.

Smears were interpreted based on the presence of acantholytic cells / nuclear changes in squamous cells / presence of inclusions / multinucleate giant cells, along with the predominant inflammatory cell type. Of these 62 Tzanck smears, 29 had histopathological correlation. Formalin fixed skin biopsies received were processed routinely and stained with $\mathrm{H} \& \mathrm{E}$.

\section{Result}

Out of 62 cases studied, 36 (58.1\%) were female and 26 (41.9\%) were male, with a M:F ratio $1: 1.38$. The youngest 
patient was 7 yrs old, the oldest being 91yrs. Maximum number of patients were in age group 61-70yrs, with a mean age of 49.6 years. A younger mean age was seen in infectious cases ( 40.7 years) as compared to non-infectious cases (58.5).

Lesions in $27.4 \%$ cases involved the entire body, followed by extremities as the commonest site. Genital involvement and a dermatomal distribution of lesions was seen predominantly in herpes cases. Oral mucosa was involved in 2 cases of herpes and one of pemphigus vulgaris. Head $\&$ neck involvement was seen in both cases of pemphigus foliaceous and one case of herpes.

Herpes was the most frequent lesion, followed by bullous pemphigoid and pemphigus. One case was of molluscum contagiosum. Tzanck smear in the remaining 13 cases was non-diagnostic. (Table 1)

All 11/11 Pemphigus cases showed acantholytic cells (Fig 1), while $6 / 25$ of the herpes cases showed acantholytic cells. In addition, all herpes cases showed multinucleated giant cells with margination of chromatin giving ground glass appearance plus 7 of them also showed intranuclear inclusions (Fig 2). One case of molluscum contagiosum showed intracytoplasmic eosinophilic inclusions with peripherally pushed nuclei (Fig 3).

Neutrophils were the predominant inflammatory cells in herpes and pemphigus whereas eosinophils were the predominant inflammatory cells in bullous pemphigoid.

Of the 62 cases 29 had histopathological diagnosis. Of these 6/29 (20.7\%) cases were pemphigus group with 4 pemphigus vulgaris, 1 pemphigus vegetans and 1 pemphigus foliaceous, showing acantholytic cells with or without inflammatory cells in the blister cavity along with tombstone basal layer (Fig 4). All pemphigus vulgaris and pemphigus vegetans cases showed intraepidermal blister (Fig 4), while pemphigus foliaceous showed subcorneal separation. Histopathology was available in 17 bullous pemphigoid cases which showed subepidermal blister with eosinophils as the predominant inflammatory cells in the blister cavity (Fig 5). Biopsy done in one case each of herpes showed multinucleate giant cells and neutrophils (Fig 2), while molluscum contagiosum showed intra cytoplasmic eosinophilic and basophilic inclusion bodies (Fig 3).

Remaining 4 cases included one each of bullous lupus erythematosus, contact dermatitis, chronic eczema and lupus vulgaris which were not able to diagnose on cytology.

Cytology showed clinical corroboration in 48 (77.4\%) cases. $25(52.1 \%)$ had viral infections and $23(47.9 \%)$ had autoimmune vesiculobullous disorders.

One case clinically suspected to be herpes simplex was diagnosed as molluscum contagiosum on cytology. The remaining 13 cases (20.9\%), cytologically showed only non-specific inflammatory cells and were non-diagnostic.

The 13 cases which were nondiagnostic on cytology were diagnosed histopathologically, as:

- 5 cases were bullous pemphigoid,

- 1 case each of pemphigus vulgaris, pemphigus foliaceous, pemphigus vegetans, herpes simplex, lupus vulgaris, bullous lupus erythematosus, chronic eczema and contact dermatitis.

Distinction between various types of Pemphigus was not possible on cytology. However, histopathology by showing blister with differing levels of separation helps in the typing.

All BP cases showed eosinophils as the predominant inflammatory cells, without specific cytological features. When these findings are clubbed with the clinical features, it enables the diagnosis. Histopathology with the subepidermal blisters containing many eosinophils is confirmatory.

In 25/26 cases of herpes cytological findings alone were sufficient to make a diagnosis with only $1 / 26$ case requiring confirmatory histopathological diagnosis.

One case of molluscum contagiosum diagnosed cytologically was confirmed on histopathology. Cytohistopathological correlation showed an overall sensitivity of $79 \%$ with $96.3 \%$ sensitivity in viral lesions $84.6 \%$ in pemphigus. Thus, emphasizing the role of Tzanck smears as a rapid diagnostic tool for vesiculobullous disorders.

Table 1: Distribution of various lesions on cytology.

\begin{tabular}{|c|c|}
\hline Cytological Diagnosis & No. of Cases \\
\hline Herpes & $25(40.3 \%)$ \\
\hline Bullous pemphigoid & $12(19.3 \%)$ \\
\hline Pemphigus & $11(17.7 \%)$ \\
\hline Molluscum contagiosum & $1(1.6 \%)$ \\
\hline Non-Diagnostic & $13(20.9 \%)$ \\
\hline Total & $\mathbf{6 2 ( 1 0 0 \% )}$ \\
\hline
\end{tabular}


Table 2: Cyto-histopathological correlation.

\begin{tabular}{|c|c|c|c|}
\hline Cytological Diagnosis & No. of Cases & Concordance & Discordance \\
\hline Herpes & 25 & - & - \\
\hline Bullous Pemphigoid & 12 & 12 & 0 \\
\hline Pemphigus & 11 & 11 & 0 \\
\hline Molluscum contagiosum & 1 & 1 & 0 \\
\hline Non-Diagnostic & 13 & 0 & $\begin{array}{c}13 \text { (5-BP, 1-PV, 1-PF, 1-PVg, 1-LV, } \\
\text { 1-BLE 1-CD, 1-CE, 1-HS) }\end{array}$ \\
\hline
\end{tabular}

(BP-Bullous Pemphigoid; PV-Pemphigus Vulgaris; PF- Pemphigus Foliaceous; PVg- Pemphigus Vegetans; LV- Lupus Vulgaris; CDContact Dermatitis; CE- Chronic Eczema; HS- Herpes Simplex).

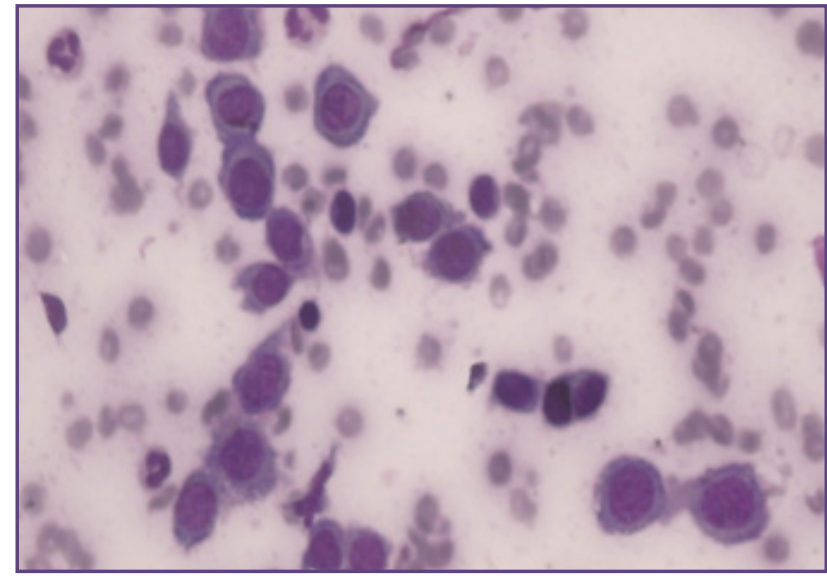

Fig. 1: Tzanck smear showing acantholytic cells (Pap, 400x).

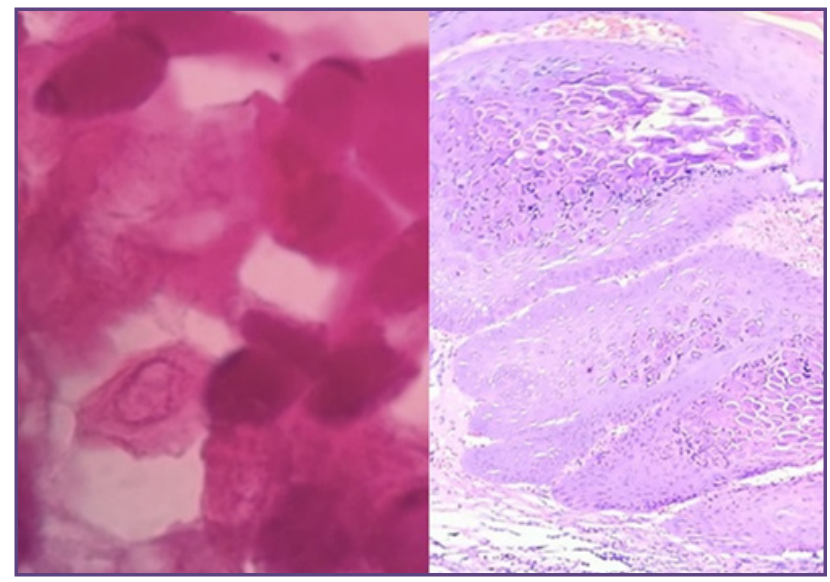

Fig. 3: Left - Tzanck smear showing eosinophilic intra cytoplasmic inclusions with pushed nucleus [Molluscum bodies] (H\&E, 400x); Right - HPE showing eosinophilic and basophilic intra cytoplasmic inclusions [Molluscum bodies] (H\&E, 200x).

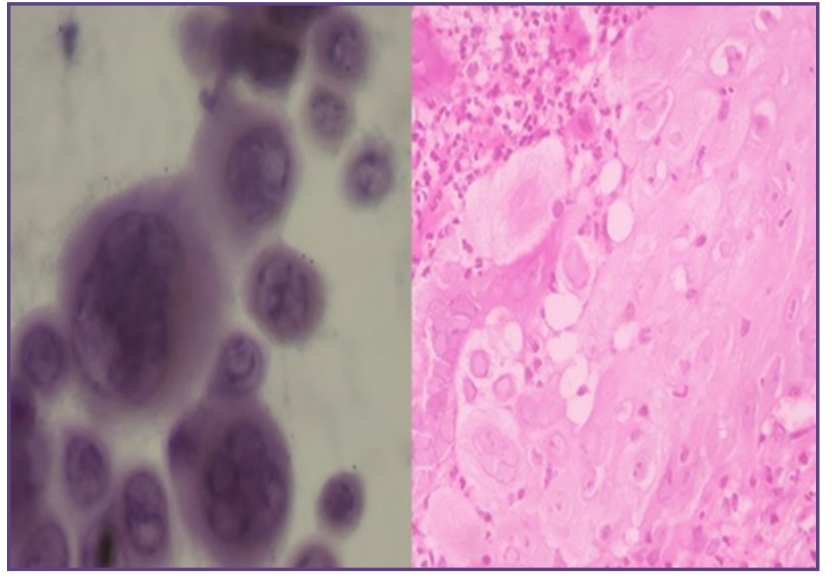

Fig. 2: Left - Tzanck smear showing multinucleation with margination of chromatin giving a ground glass appearance (Pap, 400x); Right - HPE showing multinucleation, ballooning degeneration and intranuclear inclusion (H\&E, 400x).

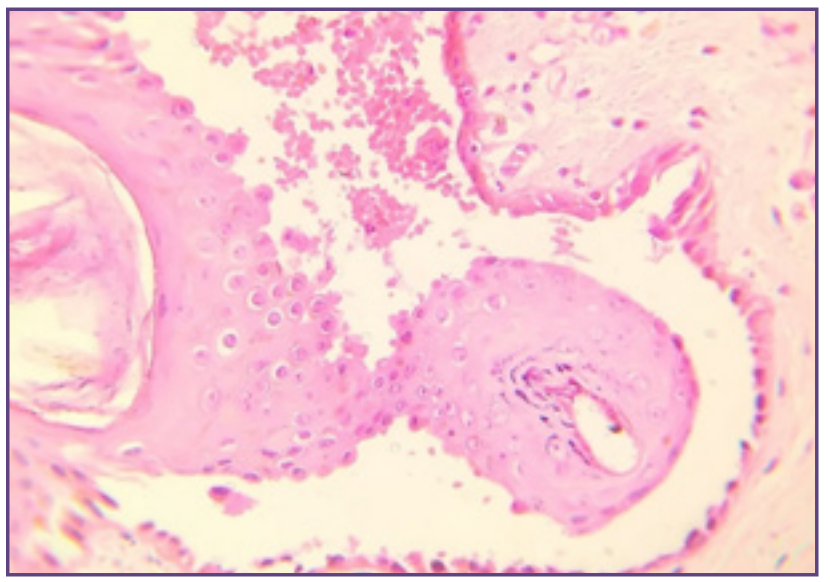

Fig. 4: HPE - Intraepidermal blister with cavity containing acantholytic cells, inflammatory cells and tombstone appearance (H\&E, 400x). 


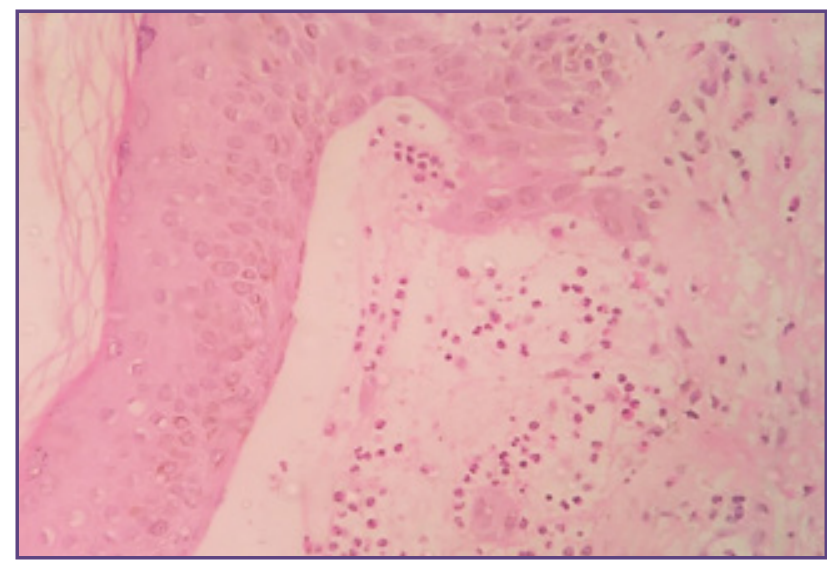

Fig. 5: HPE showing subepidermal blister with abundant eosinophils in blister cavity (H\&E, 400x).

\section{Discussion}

In the present study, the mean age of presentation was 49.6 years and females were affected more frequently than males. This was similar to study by Mittal $\mathrm{H}$ et al. ${ }^{[8]}$

Of the 62 cases, herpes was the most frequent diagnosis with $26 / 62$ (42\%) cases followed by bullous pemphigoid with 17 cases, 12 of pemphigus vulgaris and one each of pemphigus foliaceous, pemphigus vegetans, lupus vulgaris, bullous lupus erythematosus, chronic eczema and contact dermatitis. This distribution of disorders was similar to Khadse et al and Krishnamurthy et al..$^{[9,10]}$

In herpes cases Tzanck smear showed a positivity of $96 \%$ for multinucleate giant cells. Intra nuclear inclusion bodies which are characteristic of herpetic infections but are difficult to detect. In this study intranuclear inclusion bodies were identified only in $28 \%$ of the cases. One case did not show any of the above-mentioned features on cytology and was non diagnostic, however, histopathology showed multinucleate cells with ballooning degeneration which were diagnostic. These observations were comparable to Sabir et al and Durdu et al. ${ }^{[11,12]}$

As per Solomon A.R et al and Sabir et al, Tzanck smear is not helpful in differentiating between varicella and herpes infections and the distinction can be made with clinical correlation. ${ }^{[6,11]}$ Our study is in line with the same observation.

In the current study Tzanck smear showed $80 \%$ positivity for acantholytic cells in suspected cases of pemphigus. Differentiating the types of pemphigus was not possible on cytology. Histopathology by revealing varying levels of separation of epidermis helps in distinguishing the types. Intraepidermal blister seen in pemphigus vulgaris and subcorneal in pemphigus foliaceous. Similar findings were reported in other studies as well (Khadse et al, Sabir et al and Deepti S P et al)..$^{[8,11,13]}$
According to previous studies ${ }^{[2,3,12]}$ Tzanck smears in bullous pemphigoid are non-specific. Cytology shows a scarcity of epithelial cells along with eosinophil inflammatory infiltrate. We also noted similar findings, however when cytological findings are combined with clinical features possibility can be suggested. On histopathology subepidermal blister with cavity containing eosinophils is noted. Arundhati $\mathrm{S}$ et al and Heera et al reported similar findings. ${ }^{[14,15]}$

The overall sensitivity of Tzanck smear was $79 \%$ in our study which was slightly lower than that of others. ${ }^{[8,11,12]}$

\section{Conclusion}

As per our observations we can conclude that Tzanck smear is a rapid, easy and reliable tool for evaluating and diagnosing vesiculobullous lesions of the skin.

Tzanck smear cytology by showing parallel cytomorphological characters with skin biopsy can reduce the need of the skin biopsy in most of the vesiculobullous skin lesions.

Tzanck smear acts as a rapid aid to establish a precise clinical diagnosis in vesiculobullous skin lesions and has its own significance in cytodiagnosis. In addition, taking a Tzanck smear causes minimal trauma or discomfort to the patient allowing it to be easily performed.

Although not a substitute for routine histopathological evaluation, Tzanck smear is a useful tool to establish the clinical diagnosis with ease and rapidity, functioning as an adjunct to the standard techniques in diagnosing vesiculobullous disorders. This study may be taken as reinforcement to other studies that have already established the utility of Tzanck smear as a cytodiagnostic technique in the field of dermatology.

\section{Acknowledgements}

We would like to acknowledge Dr. Sharath and Dr. Nandini, Dept. of Dermatology for providing clinical material required for the study and thank our HOD (Dept. of Pathology), Dr. Suguna B.V for constant support.

\section{Funding}

None

\section{Competing Interests}

None declared

\section{References}

1. Tzanck A. Le cytodiagnostic immediate en dermatologie. Bull Soc Fr Dermatol Syph 1947;7:68 (Quoted from Barr RJ, Irvine CA. Cutaneous cytology. J Am Acad Dermatol 1984;10:163-80) 
2. Barr RJ. Cutaneous cytology. J Am Acad Dermatol 1984;10:163-80.

3. Ruocco V, Ruocco E. Tzanck smear, an old test for the new millennium: when and how? Int J Dermatol 1999;38:830-4.

4. Graham JH, Bingul O, Burgoon CF Jr. Cytodiagnosis of inflammatory dermatosis: Pitfalls in evaluation of smears. Arch Dermatol 1963;87:18-27.

5. Blank H, Burgoon CF Jr. Abnormal cytology of epithelial cells in pemphigus vulgaris: A diagnostic aid. J Invest Dermatol 1952;18:213-23.

6. Solomon AR, Rasmussen JE, Weiss JS. A comparison of the Tzanck smear and viral isolation in varicella and herpes zoster. Arch Dermatol 1986;122:282-5.

7. Wu H, Schapiro B, Harrist TJ. Noninfectious vesiculobullous and vesiculopustular diseases. In: Elder D, Elenitsas R, Johnson BL, Murphy GF, ed. Lever's histopathology of the skin, 9th edition. Philadelphia: Lippincott Williams and Wilkins, 2005: p243-291.

8. Mittal $\mathrm{H}$ et al. A study of clinicopathologic spectrum of vesicobullous disorders. Int J Res Dermatol. 2017 Sep;3(3):355-364.

9. Khadse $\mathrm{V}$ et al. The study of vesicobullous skin lesions by Tzanck smear cytology. IP Archives of Cytology and Histopathology Research, April-June 2018;3(2):61-64.
10. Krishnamurthy $\mathrm{T}$ et al. Histopathological Study of Vesiculobullous lesions of skin. Int J Biol Med Res. 2015; 6(2): 4966-4972.

11. Sabir F, Aziz M, Afroz N, Amin SS. Clinical and cytohistopathological evaluation of skin lesions with special reference to bullous lesions. Indian J Pathol Microbiol 2010; 53(10): 41-46.

12. Durdu M, Baba M,Sekin D. The value of Tzanck smear test in diagnosis of erosive, vesicular, and pustular skin lesions. J Am Acad Dermatol. 2008; 59: 958-64.

13. Deepti S, Sulakshana MS, Manjunatha YA, Jayaprakash HT. A histomorphological study of bullous lesions of skin with special reference to immunofluorescence. Int J Curr Res Aca Rev. 2015; 3(3): 29-51.

14. Arundhathi S, Raghunatha S, Mahadeva KC. A Crosssectional study of clinical, histopathological and direct immunofluorescence spectrum of vesiculobullous disorders. J Clin Diagn Res. 2013; 7(12); 2788-92.

15. Heera K P, Anoop T V, Ajaya Kumar S, Robins K, Rajiv S. The significance of tzanck smear in evaluation of vesiculo bullous skin lesions in correlation with clinical diagnosis - a cross sectional study. International Journal of Contemporary Medical Research 2017;4(2):337-340.

*Corresponding author:

Dr. Hemalata M, Professor, Dept. of Pathology, KIMS College, Banashankari Stage II Bengaluru (Karnataka) 560070 INDIA

Phone: +919986151903

Email: hemalatajreddy@gmail.com

Date of Submission : 22/01/2020

Financial or other Competing Interests: None. 